\title{
Maf1 regulation
}

\section{A model of signal transduction inside the nucleus}

Yuehua Wei and X.F. Steven Zheng*

Department of Pharmacology and Cancer Institute of New Jersey; UMDNJ-Robert Wood Johnson Medical School; Piscataway, NJ USA

Key words: Maf1, target of rapamycin (TOR), RNA polymerase III, ribosomal DNA (rDNA), transfer RNA (tRNA), nucleolus

Abbreviations: TOR, the target of rapamycin; TORC1, TOR complex 1; TORC2, TOR complex 2; PI3K, phosphoinositide 3-kinase; NLS, nuclear localization sequence; AA, amino acid; Pols, RNA polymerases; rRNA, ribosomal RNA ; rDNA, ribosomal DNA; tRNA, transfer RNA; PKA, protein kinase A; PP2A, protein phosphatase $2 \mathrm{~A}$

Submitted: $11 / 30 / 09$

Revised: 01/06/10

Accepted: 01/07/10

Previously published online: www.landesbioscience.com/journals/ nucleus/article/11179

*Correspondence to: X.F. Steven Zheng; Email: zhengst@umdnj.edu
$\mathbf{R}^{\mathrm{s}}$ NA polymerase III (Pol III) is responsible for the synthesis of $5 \mathrm{~S}$ ribosomal RNA (rRNA) and transfer RNAs (tRNAs) essential for protein synthesis and cell growth. Pol III is tightly controlled by growth signals such as nutrients and deregulation of Pol IIIdependent transcription can lead to oncogenic transformation. In response to extracellular stimuli, the target of rapamycin complex 1 (TORC1) regulates Pol III activity through Maf1, a key conserved Pol III repressor. Recent studies have unraveled intricate mechanisms by which Maf1 activity is controlled at multiple levels, including nuclear transport and phoshorylation at specific chromatin loci. These studies suggest an emerging mode of gene regulation by extracellular signals inside the nucleus.

\section{Pol III is a Major Target of Growth Signals}

Eukaryotic cells contain three major RNA polymerases: Pol I, II and III. Pol I is specifically required for production of ribosomal RNA including 18S, $5.8 \mathrm{~S}$ and $25 \mathrm{~S}$ rRNAs; Pol II transcribes all the mRNAs and a few of the small nuclear RNAs; Pol III is dedicated to synthesis of the $5 \mathrm{~S}$ subunit of rRNA, tRNAs and a number of short RNAs that play both structural and regulatory roles. ${ }^{1,2}$ Of the three major eukaryotic RNA polymerases, Pol III is the largest one that contains over 17 subunits with an overall 0.7 MDa molecular weight. ${ }^{3}$ rRNAs and tRNAs are essential components of the protein synthesis machinery, which account for up to $95 \%$ of overall transcripts in a cell. ${ }^{4}$ Therefore,
Pol I and Pol III are tightly regulated by nutrient signals. Deregulation of Pol I- and Pol III-dependent transcription has been a hallmark of numerous cancer cells and has attracted tremendous attentions in recent years. Significantly, overexpression of Pol III products is oncogenic, ${ }^{5}$ suggesting a causal role of Pol III in cancer formation. Pol III transcription machineries are targeted directly by several oncogene products such as Erk and c-Myc, or tumor suppressors such as RB and $\mathrm{p} 53$, demonstrating the critical role of Pol III in tumorigenesis. ${ }^{4}$

Nutrient signals are relayed to Pol III through a conserved PI3K-related kinase (PIKK) called TOR (target of rapamycin). TOR proteins are highly conserved from budding yeast (Tor1 and Tor2) to mammals (mTOR) in both structure and function. ${ }^{6}$ TOR functions through two conserved complexes TORC1 and TORC2. ' TORC1 is a nutrient sensing complex that coordinates the activities of all three Pols for ribosome biogenesis and tRNA synthesis. ${ }^{8}$ In both yeast and mammalian cells, TORC1 inhibition by rapamycin or nutrient starvation elicits a strong inhibition on synthesis of $5 \mathrm{~S}$ rRNA and tRNAs, ${ }^{9-14}$ suggesting that Pol III is a major target of growth signals. Regulation of Pol III by TORC1 requires a conserved transcription factor Maf1. Recent progresses begin to elucidate the intricate mechanisms by which Maf1 is regulated by TORC1.

\section{Maf1 is a Conserved Mediator of Growth Signals to Pol III}

The Maf1 protein is a hydrophilic protein conserved from yeast to human. It 


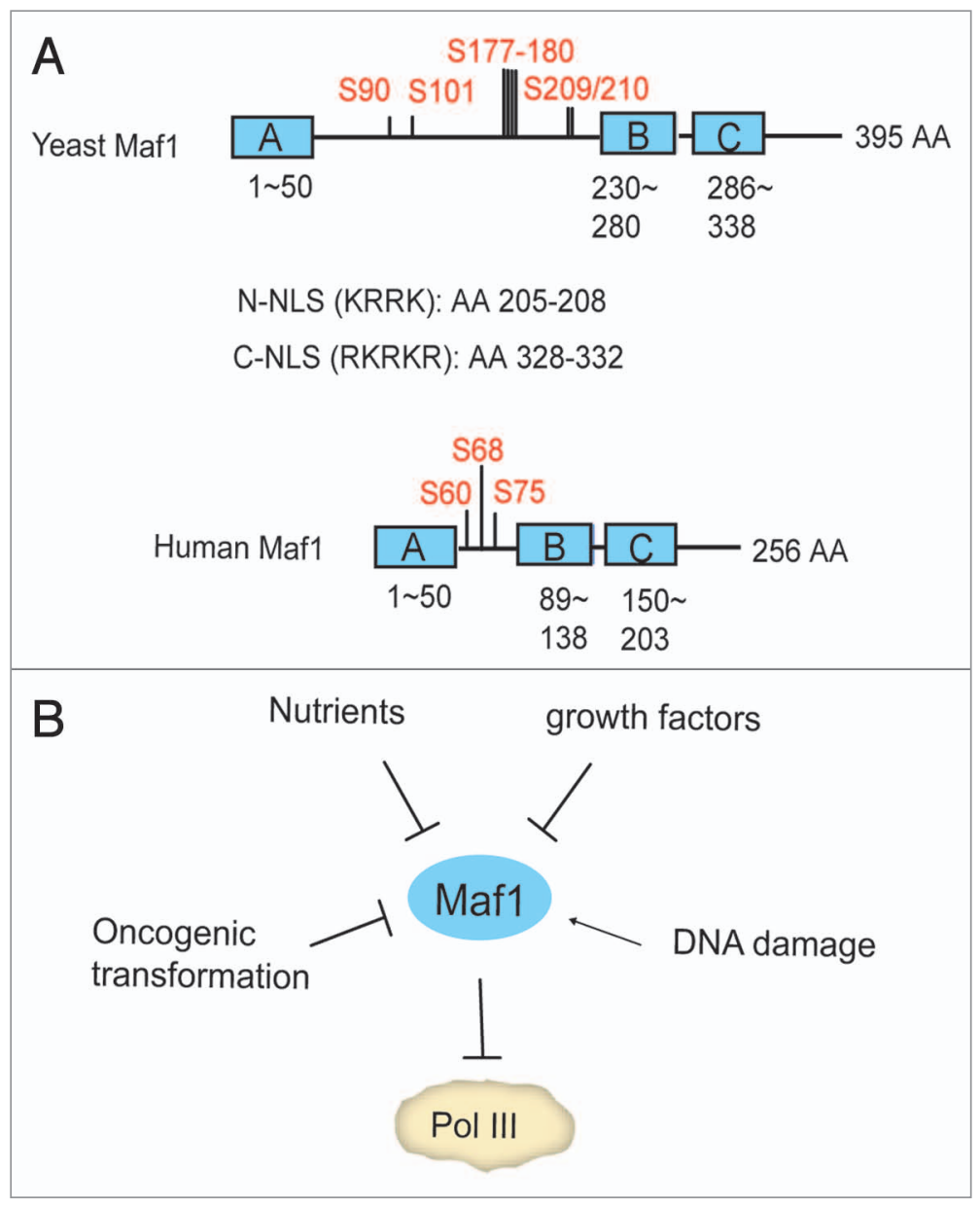

Figure 1. Maf1 is a central mediator of extracellular signals for Pol III regulation. (A) Protein structure organization of yeast and human Maf1 proteins. Maf1 contains three evolutionally conserved domains ( $A, B$ and $B$ boxes). The amino acid number below each domain indicates the approximate position. Yeast Maf1 contains 395 amino acids rich in Serine. Two conserved nuclear localization sequences (N-NLS and C-NLS) are shown below. Human Maf1 is 256 amino acids in size but lacks a canonical NLS. Both yeast and human Maf1 are phosphorylated at multiple phosphoserine residues in between $A$ box and $B$ box. (B) Various signaling pathways converge on Maf1 to regulate Pol III-dependent transcription. Maf1 is a Pol III repressor. Nutrients, growth factors and oncogenic mutations promote Pol III-dependent transcrirption through inhibiting Maf1 activity. In contrast, DNA damage pathway represses Pol III by activating Maf1.

contains three signature domains: A, B and $\mathrm{C}$ boxes. ${ }^{15}$ Although Maf1 from budding yeast and human are variable in some regions, the three signature domains are highly conserved among Maf1 proteins but not found in any other polypeptides (Fig. 1A). Yeast Maf1 contains two conserved nuclear localization sequences (NLS) at the $\mathrm{N}$ - and C-termini. ${ }^{16}$ In contrast, human Maf1 does not contain any recognizable NLS. Recent studies have identified several phosporylation sites of both yeast and human Maf1. The yeast Maf1 phosphorylation sites shown in Figure 1A are targeted by Sch9 and/ or protein kinase $A$ (PKA). In addition, phosphorylation sites were reported on human Maf1 through phospho-proteomic studies but their functional significance has not yet been determined. Interestingly, most of the phosphorylation sites occur in the region between $\mathrm{A}$ and $\mathrm{B}$ boxes, suggesting that this region is targeted by regulatory kinases and phosphatases.

Maf1 is first identified in budding yeast by a mutation that decreases the efficiency of SUP11 (gene encoding tRNA ${ }^{\mathrm{Tyr}}$ ) to suppress the nonsense mutation ade21. ${ }^{15}$ MAF1 deletion specifically elevates tRNAs and $5 \mathrm{~S}$ rRNA by both in vivo and in vitro transcription analysis. ${ }^{16}$ Moreover, Maf1 protein interacts with the Pol III transcription machinery including the Rpc160 subunit of Pol III, and a regulatory subunit Brf1. These data suggest Maf1 as a Pol III repressor. Subsequent studies reveal a broad range of signaling pathways including nutrient sensing and DNA damage pathways that impinge on Maf1. ${ }^{17}$ Maf1 is therefore a central module for Pol III regulation. Mammalian Maf1 is also an essential mediator of Pol III regulation by nutrients and growth factors, and similarly regulated by binding to Pol III, as demonstrated in several cell types including heterozygous embryonic stem cell. ${ }^{11-14}$ Other than 5S rRNA and tRNAs, mammalian Maf1 also represses transcription of small regulatory RNAs such as U6 and 7SK RNAs, suggesting that Maf1 affects Pol III-dependent transcription globally. Importantly, Maf1 overexpression inhibits oncogenic transformation, ${ }^{13}$ likely due to Maf1's ability to restrain Pol III-dependent transcription.

\section{The Control of Maf1 Subcellular Localization}

Similar to many transcriptional factors, Maf1 is regulated at the level of cytoplasmto-nucleus transport. Maf1 is distributed in both the nucleus and cytoplasm in certain yeast strains. It becomes further enriched the nucleus upon rapamycin treatment or nutrient starvation. ${ }^{18-20}$ The nucleus-to-cytoplasm transport is regulated by PKA- and Sch9-dependent phosphorylation. PKA phosphorylation of yeast Maf1 includes two residues adjacent to the conserved N-terminal NLS (Fig. 1A). It has been proposed that PKA promotes Maf1 cytoplasmic localization through "switching off" the N-terminal NLS by phosphorylation. ${ }^{18}$ Sch9 targets largely the same Maf1 residues for phosphorylation, consistent with its role in regulation of Maf1 nuclear transport. ${ }^{21,22}$ However, PKA- or Sch9-dependent phosphorylation is not sufficient to fully retain Maf1 in the cytoplasm, suggesting that both PKA and Sch9 are only partially involved. ${ }^{21,23}$ Regulation of Maf1 localization is achieved by coordination of both the N-terminal and C-terminal NLS. Recent studies also revealed Msn5 as a nuclear exportin for Maf1. ${ }^{24}$ In certain other yeast strains, Maf1 is constitutively 
nuclear, which is determined by SSD $1 .^{25}$ $S S D 1$ was initially identified by a mutant to suppress sit4 deletion, ${ }^{26}$ which plays a key role in mediating TORC1 signals. Yeast cell lacking SSD1 gene is hypersensitive to rapamycin treatment. ${ }^{25}$ Moreover, Ssd1 is involved in Pol I- and Pol III-dependent transcription. ${ }^{27}$ These observations suggest that Ssd1 is intimately involved in transmitting TORC1 signals to Maf1.

Despite the initial enthusiasm, the nucleus-to-cytoplasm transport turns out to be dispensable for Maf1 regulation. In yeast cells lack of the nuclear exportin Msn5, or in W303a cells in which Maf1 is constitutively nuclear, Maf1 phosphorylation and Pol III-dependent transcription remain fully regulated by TORC1, 18,24,25 suggesting that the essential steps for Maf1 control occur in the nucleus. This is consistent with the observations that without PKA- and Sch9-dependent phosphorylation, Maf1 basal phosphorylation and activity remain regulated by TORC1..$^{21}$ The predominant nuclear localization of Maf1 in mammalian cells lends further support for this idea. ${ }^{12}$ Interestingly, the yeast Maf1 is also dynamically distributed within the nucleus. It normally stays in the nucleoplasm but is absent from the nucleolus. However, Maf1 translocates into the nucleolus upon rapamycin treatment or starvation. ${ }^{21,25}$ Nucleolus is the site for ribosome biogenesis, in which $5 \mathrm{~S}$ rDNA is localized. The nucleoplasmto-nucleolus transport of Maf1 provides a new mechanism for Maf1 regulation within the nucleus. Since mammalian Maf1 largely resides in the nucleus, it is conceivably subjected to the same nucleoplasm-to-nucleolus regulation.

\section{TORC1 Regulates Maf1 at 5S rDNA Promoter}

How is Maf1 regulated within the nucleus? Maf1 is known to inhibit Pol III-dependent transcription through direct binding to target genes such as tRNA genes (tDNAs) and $5 \mathrm{~S}$ rRNA gene (rDNA). ${ }^{19,20}$ TORC1 shuttles between the cytoplasm and nucleus, and binds to rDNA promoters. ${ }^{28}$ These activities of TORC1 are essential for its regulation of Pol I and Pol III-dependent transcription ${ }^{28}$

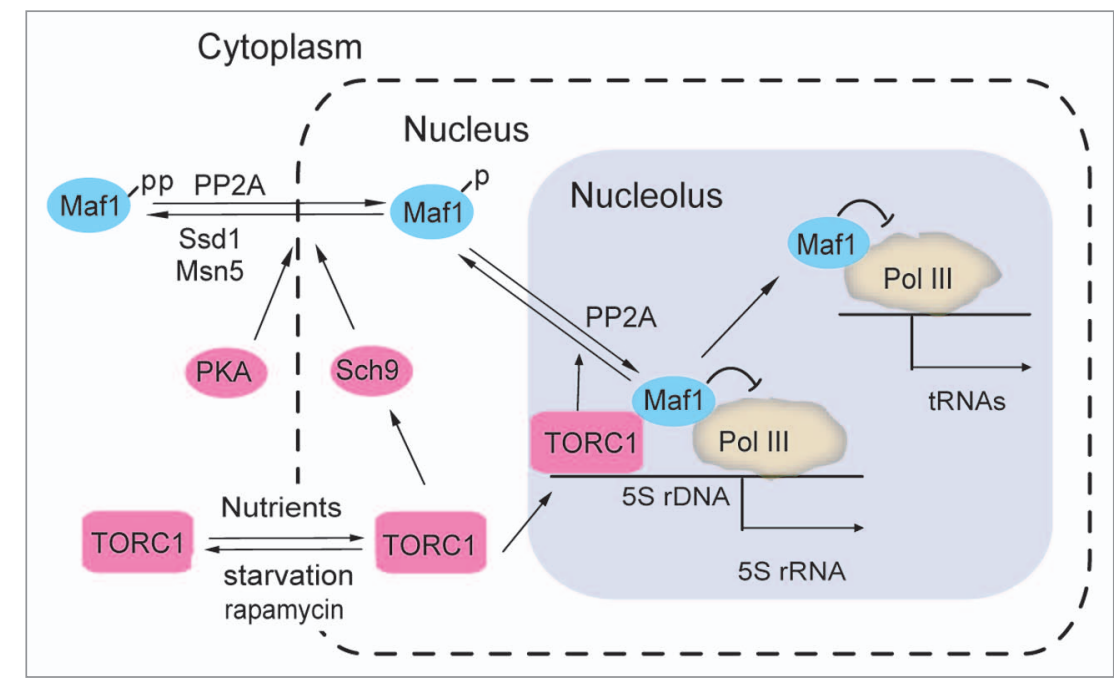

Figure 2. Maf1 is essentially controlled by TORC1 in the nucleolus. TORC1 senses the intracellular nutrient availability. With ample nutrients, TORC1 translocates into the nucleus and binds to $5 \mathrm{~S}$ rDNA chromatin to promote Pol III-directed RNA synthesis. At the 5S rDNA chromatin site, TORC1 regulates Maf1 nucleolar localization and Pol III binding through phosphorylation, which is essential for Pol III-dependent transcription. Sch9, PKA Ssd1 and Msn5 are involved in controlling Maf1 nucleus-to-cytoplasm transport, which provides an optimal but not essential regulation of Maf1. tRNA synthesis is indirectly regulated by TORC1 through controlling Maf1 nucleolar localization. Maf1 nucleolar localization thus serves to coordinate transcription of both rRNAs and tRNAs.

as well as Maf1 phosphorylation and regulation. ${ }^{25}$ Moreover, TORC1 phosphorylates recombinant Maf1 in vitro in a rapamycin-sensitive manner. These studies strongly suggest that TORC1 is an essential nuclear kinase for Maf1. These observations further suggest that TORC1 regulates Maf1 at the $5 \mathrm{~S}$ rDNA promoter site, preventing Maf1 accumulation at the nucleolus and inhibition of $5 \mathrm{~S} \mathrm{rDNA}$ transcription (Fig. 2). Phosphatase PP2A, also acting downstream of TORC1, is known to dephosphorylate Maf1. ${ }^{19}$ The reversible phosphorylation-dephosphorylation by TORC1 and PP2A is also common to other TORC1 substrates such as S6K1 and Gln3. ${ }^{29-31}$ The phosphorylation site(s) regulated by TORC1 remains to be identified, but it is unlikely to overlap Sch9- or PKA-dependent sites since mutation of these sites does not affect TORC1 regulation of Maf1 basal phosphorylation and activity. ${ }^{21}$ Currently, it is not known whether mTORC1 regulates Maf1 similarly in mammalian cells. Interestingly, mTORC1 is found in the nucleus, ${ }^{32,33}$ and bound to the promoters of Pol I- and Pol III-transcribed genes (Tsang et al. unpublished observations), suggesting that the role of mTORC1 in Maf1 regulation is conserved.

\section{The Regulation of tRNA Synthesis by TORC1}

Besides $5 \mathrm{~S}$ rRNA, tRNAs are also similarly regulated by TORC1 through Maf1, which requires TORC1 to associate with chromatin. However, no significant TORC1 binding can be detected at tRNA loci in yeast. ${ }^{25}$ TORC1 therefore appears to modulate tRNA synthesis indirectly. Then how are tRNA genes regulated by TORC1 through Maf1? Interestingly, despite that 274 yeast tRNA genes are scattered throughout different chromosomes, it was found that they are organized inside the nucleolus. ${ }^{34}$ However, precisely why tRNA genes are aggregated into the nucleolus is not clear. One possible reason is that tRNA processing occurs in the nucleolus. ${ }^{35}$ Therefore, it is much more efficient if tRNA transcription takes place in the same location. Another explanation for tRNA genes to cluster in the nucleolus regulation is to provide efficient control by modulating Maf1 nucleolar localization (Fig. 2). Under normal growth conditions, Maf1 is phosphorylated by TORC1, which causes Maf1 to be excluded from the nucleolus, preventing Maf1 from binding to promoters of tRNA genes and inhibiting tRNA synthesis. Conversely, nutrient 
starvation inhibits TORC1-dependent Maf1 phosphorylation, leading to Maf1 nucleolar localization and inhibition of tRNA synthesis. In addition, this nucleolar clustering may provide coordination of ribosome biogenesis and tRNA synthesis, which could be important for balancing different components involved in protein synthesis.

\section{Conclusions and Prospects}

By using budding yeast as a model, recent studies identify Maf1 as a Pol III inhibitor that is controlled by TORC1 through phoshorylation and nucleolar localization (Fig. 2). Interestingly, Maf1 is also regulated through nucleus-to-cytoplasm transport by Sch9 and/or PKA, demonstrating a strict regulatory network by several kinases at multiple levels. Despite the current paradigm that gene regulation through nuclear transport of specific transcription factors, this mode of regulation is not essential for Maf1 activity. In contrast, Maf1 regulation by the nutrient sensing kinase TORC1 inside the nucleus and at the $5 \mathrm{~S}$ rDNA is essential. This fits into an emerging mode of gene regulation by which transcription factors are directly regulated inside the nucleus or at the chromatin sites, which could provide rapid responses and efficient gene regulation. ${ }^{36}$ Given the significance of Maf1 in Pol III-dependent transcription, it is likely that future studies will unravel other mechanisms for Maf1 regulation. For example, since DNA damage signal and secretion pathway also target Maf1, there could involve other kinases and phosphatases. Alternatively, such signals may regulate Maf1 through other post-translational mechanisms such as protein degradation. TORC1, Maf1 and Pol III are highly conserved from yeast to humans. Overexpression of mammalian Maf1 is known to restrain cellular transformation in vitro, suggesting that Maf1 is potentially a tumor suppressor. It would be of especial interest to investigate whether Maf1 is deregulated in human cancers and targeting Maf1 regulation is feasible for anticancer drug development. Delineation of the pathways that regulate Maf1 in both human cells and model organisms such as yeast may prove important for therapeutic intervention of human diseases.

\section{Acknowledgements}

This work was supported by NIH grant R01-CA123391 (X.S.Z.).

\section{References}

1. Moss T, Stefanovsky VY. At the center of eukaryotic life. Cell 2002; 109:545-8.

2. Warner JR. The economics of ribosome biosynthesis in yeast. Trends Biochem Sci 1999; 24:437-40.

3. Jasiak AJ, Armache KJ, Martens B, Jansen RP, Cramer P. Structural biology of RNA polymerase III: subcomplex C17/25 X-ray structure and 11 subunit enzyme model. Mol Cell 2006; 23:71-81.

4. White RJ. RNA polymerases I and III, growth control and cancer. Nat Rev Mol Cell Biol 2005; 6:6978.

5. Marshall L, White RJ. Non-coding RNA production by RNA polymerase III is implicated in cancer. Nat Rev Cancer 2008; 8:911-4.

6. Wullschleger S, Loewith R, Hall MN. TOR signaling in growth and metabolism. Cell 2006; 124:471-84.

7. Loewith R, Jacinto E, Wullschleger S, Lorberg A, Crespo JL, Bonenfant D, et al. Two TOR complexes, only one of which is rapamycin sensitive, have distinct roles in cell growth control. Mol Cell 2002; 10:457-68.

8. Mayer C, Grummt I. Ribosome biogenesis and cell growth: mTOR coordinates transcription by all three classes of nuclear RNA polymerases. Oncogene 2006; 25:6384-91.

9. Powers T, Walter P. Regulation of ribosome biogenesis by the rapamycin-sensitive TOR-signaling pathway in Saccharomyces cerevisiae. Mol Biol Cell 1999; 10:987-1000.

10. Zaragoza D, Ghavidel A, Heitman J, Schultz MC. Rapamycin induces the $G_{0}$ program of transcriptional repression in yeast by interfering with the TOR signaling pathway. Mol Cell Biol 1998; 18:4463-70.

11. Goodfellow SJ, Graham EL, Kantidakis T, Marshall L, Coppins BA, Oficjalska-Pham D, et al. Regulation of RNA polymerase III transcription by Maf1 in mammalian cells. J Mol Biol 2008; 378:481-91.

12. Rollins J, Veras I, Cabarcas S, Willis I, Schramm L. Human Maf1 negatively regulates RNA polymerase III transcription via the TFIIB family members Brf1 and Brf2. Int J Biol Sci 2007; 3:292-302.

13. Johnson SS, Zhang C, Fromm J, Willis IM, Johnson DL. Mammalian Maf1 is a negative regulator of transcription by all three nuclear RNA polymerases. Mol Cell 2007; 26:367-79.

14. Reina JH, Azzouz TN, Hernandez N. Maf1, a new player in the regulation of human RNA polymerase III transcription. PLoS One 2006; 1:134.

15. Ciesla M, Boguta M. Regulation of RNA polymerase III transcription by Maf1 protein. Acta Biochim Pol 2008; 55:215-25.

16. Pluta K, Lefebvre O, Martin NC, Smagowicz WJ, Stanford DR, Ellis SR, et al. Maflp, a negative effector of RNA polymerase III in Saccharomyces cerevisiae. Mol Cell Biol 2001; 21:5031-40.

17. Upadhya R, Lee J, Willis I. Maf1 is an essential mediator of diverse signals that repress RNA polymerase III transcription. Mol Cell 2002; 10:1489-94.

18. Moir RD, Lee J, Haeusler RA, Desai N, Engelke DR, Willis IM. Protein kinase A regulates RNA polymerase III transcription through the nuclear localization of Maf1. Proc Natl Acad Sci USA 2006; 103:15044-9

19. Oficjalska-Pham D, Harismendy O, Smagowicz W, Gonzalez de Pered A, Boguta M, Sentenac A, et al. General repression of RNA polymerase III transcription is triggered by protein phosphatase type 2A-mediated dephosphorylation of Maf1. Mol Cell $2006 ; 22: 623-32$.
20. Roberts D, Wilson B, Huff J, Stewart A, Cairns B. Dephosphorylation and genome-wide association of Maf1 with Pol III-transcribed genes during repression. Mol Cell 2006; 22:633-44.

21. Wei Y, Zheng XF. Sch9 partially mediates TORC1 signaling to control ribosomal RNA synthesis. Cell Cycle 2009; 8:4085-90.

22. Huber A, Bodenmiller B, Uotila A, Stahl M, Wanka $S$, Gerrits B, et al. Characterization of the rapamycinsensitive phosphoproteome reveals that Sch9 is a central coordinator of protein synthesis. Genes Dev 2009; 23:1929-43.

23. Lee J, Moir RD, Willis IM. Regulation of RNA polymerase III transcription involves $\mathrm{SCH}$ 9-dependent and $\mathrm{SCH}$ 9-independent branches of the target of rapamycin (TOR) pathway. J Biol Chem 2009; 284:12604-8.

24. Towpik J, Graczyk D, Gajda A, Lefebvre O, Boguta M. Derepression of RNA polymerase III transcription by phosphorylation and nuclear export of its negative regulator, Maf1. J Biol Chem 2008; 283:17168-74.

25. Wei Y, Tsang CK, Zheng XF. Mechanisms of regulation of RNA polymerase III-dependent transcription by TORC1. EMBO J 2009; 28:2220-30.

26. Sutton A, Immanuel D, Arndt KT. The SIT4 protein phosphatase functions in late $G_{1}$ for progression into S phase. Mol Cell Biol 1991; 11:2133-48.

27. Stettler S, Chiannilkulchai N, Hermann-Le Denmat S, Lalo D, Lacroute F, Sentenac A, et al. A general suppressor of RNA polymerase I, II and III mutations in Saccharomyces cerevisiae. Mol Gen Genet 1993; 239:169-76

28. Li H, Tsang CK, Watkins M, Bertram PG, Zheng XF. Nutrient regulates Tor1 nuclear localization and association with rDNA promoter. Nature 2006; 442:1058-61.

29. Duvel K, Broach J. The role of phosphatases in TOR signaling in yeast. Curr Top Microbiol Immunol 2004; 279:19-38.

30. Peterson RT, Desai BN, Hardwick JS, Schreiber SL. Protein phosphatase $2 \mathrm{~A}$ interacts with the $70-\mathrm{kDa}$ S6 kinase and is activated by inhibition of FKBP12rapamycinassociated protein. Proc Natl Acad Sci USA 1999; 96:4438-42.

31. Beck T, Hall MN. The TOR signalling pathway controls nuclear localization of nutrient-regulated transcription factors. Nature 1999; 402:689-92.

32. Kim JE, Chen J. Cytoplasmic-nuclear shuttling of FKBP12-rapamycin-associated protein is involved in rapamycin-sensitive signaling and translation initiation. Proc Natl Acad Sci USA 2000; 97:14340-5.

33. Zhang X, Shu L, Hosoi H, Murti KG, Houghton PJ. Predominant nuclear localization of mammalian target of rapamycin in normal and malignant cells in culture. J Biol Chem 2002; 277:28127-381.

34. Thompson M, Haeusler RA, Good PD, Engelke DR. Nucleolar clustering of dispersed tRNA genes. Science 2003; 302:1399-401.

35. Bertrand E, Houser-Scott F, Kendall A, Singer RH, Engelke DR. Nucleolar localization of early tRNA processing. Genes Dev 1998; 12:2463-8.

36. Edmunds JW, Mahadevan LC. Cell signaling. Protein kinases seek close encounters with active genes. Science 2006; 313:449-51. 